\title{
Atuação de uma liga acadêmica de cuidados à saúde da mulher durante o primeiro ano da pandemia da COVID-19
}

\author{
Performance of an women's health care academic league during the first year of the \\ COVID-19 pandemic
}

\section{Desempeño de una liga académica de cuidado de la salud de la mujer durante el primer año de la pandemia COVID-19}

Nathalia Santarato ${ }^{1 *}$, Wellery Stefany Nunes Glavina ${ }^{1}$, Amanda Maria Urei Rodrigues ${ }^{1}$, Cecília Verones Cândido de Morais ${ }^{1}$, Gleicy Kelly Felix Costa ${ }^{1}$, Julia Ferreira Cieto ${ }^{1}$, Thamíris Martins Michelon ${ }^{1}$, Juliana Cristina dos Santos Monteiro ${ }^{1}$, Ludmila de Oliveira Ruela1, Mônica Maria de Jesus Silva1.

\section{RESUMO}

Objetivo: Relatar a experiência de atuação de uma Liga Acadêmica de Cuidados à Saúde da Mulher durante 0 ano de 2020 apresentando as dificuldades e facilidades encontradas frente às atividades remotas. Relato da experiência: A Liga Acadêmica estudada pertencente a um curso de graduação em Enfermagem de uma universidade pública brasileira e ao longo do primeiro ano da pandemia da COVID-19, precisou se adaptar frente ao novo modelo de atividades. Nesse período, houve a mudança do ensino presencial para o ensino remoto com a utilização de plataformas digitais e redes sociais, o que proporcionou facilidades e dificuldades para o desempenho das atividades. Isso acarretou no alcance de público maior e mais variado, permitindo atender aos preceitos que devem ser seguidos por uma Liga Acadêmica, entretanto, o acesso às atividades dependia de maior nível de concentração e ambiente propício a isso, internet disponível e aparelhos eletrônicos, o que pode ter dificultado o desempenho da Liga. Considerações finais: A atuação da Liga Acadêmica demonstrou ser uma experiência desafiadora, porém, exitosa, que foi possível pela incorporação das Tecnologias da Informação e Comunicação e por meio da utilização de plataformas que permitiram manter ativas as atividades desenvolvidas e alcançar espaços antes pouco possíveis.

Palavras-chave: COVID-19, Pandemias, Saúde da mulher, Enfermagem, Tecnologia da informação e comunicação.

\section{ABSTRACT}

Objective: Report the experience of an Academic League for Women's Health Care during 2020, presenting the difficulties and facilities found in the face of remote activities. Experience report: The Academic League studied, belonging to an undergraduate course in Nursing at a Brazilian public university and throughout the first year of the COVID-19 pandemic, needed to adapt to the new model of activities. In this period, there was a change from face-to-face teaching to remote teaching with the use of digital platforms and social networks, which provided facilities and difficulties for the performance of activities. This resulted in the reach of a wider and more varied public, allowing to meet the precepts that must be followed by an Academic League, however, access to activities depended on a higher level of concentration and an environment conducive to this, available internet and electronic devices, the that may have hampered the League's performance. Final considerations: The performance of the Academic League proved to be a challenging but successful experience, made possible by the incorporation of Information and Communication Technologies and using of the platforms that allowed to keep the developed activities active and reach spaces that were not previously possible.

Keywords: COVID-19, Pandemics, Women's health, Nursing, Information technology.

1 Universidade de São Paulo (USP), Ribeirão Preto - SP. *E-mail: nathaliasantarato@usp.br 


\section{RESUMEN}

Objetivo: Reporte la experiencia de una Liga Académica para la Atención de la Salud de la Mujer durante 2020, presentando las dificultades y facilidades encontradas ante las actividades a distancia. Informe de experiencia: La Liga Académica en cuestión, perteneciente a un curso de Enfermería de una universidad pública brasileña y durante todo el primer año de la pandemia COVID-19, tuvo que adaptarse al nuevo modelo de actividades. Durante este período, hubo un cambio de la enseñanza presencial a la enseñanza a distancia con el uso de plataformas digitales y redes sociales, lo que brindó facilidades y dificultades para el desempeño de las actividades. Esto resultó en el alcance de un público más amplio y variado, permitiendo cumplir con los preceptos que debe seguir una Liga Académica, sin embargo, el acceso a las actividades dependía de un mayor nivel de concentración y un entorno propicio para ello, disponibilidad de internet y dispositivos electrónico, que pueden haber obstaculizado el desempeño de la Liga. Consideraciones finales: El desempeño de la Liga Académica resultó ser una experiencia desafiante pero exitosa, posibilitada por la incorporación de Tecnologías de la Información y la Comunicación y mediante el uso de plataformas que permitieron mantener activas las actividades desarrolladas y alcanzar espacios ante difícil de alcanzar.

Palabras clave: COVID-19, Pandemias, Salud de la mujer, Enfermería, Tecnología de la información.

\section{INTRODUÇÃO}

Em dezembro de 2019, foi descoberta uma nova doença infecciosa causada por um vírus da família coronavírus, o Sars-Cov-2. A doença recebeu o nome de COVID-19 e devido à sua fácil contaminação e consequente disseminação descontrolada, em pouco tempo haviam milhares de contaminados e mortos. Diante da grave situação e do aumento importânte do número de casos, no dia 11 de março de 2020 a Organização Mundial da Saúde (OMS) caracterizou a COVID-19 como uma pandemia (ORGANIZAÇÃO MUNDIAL DA SAÚDE, 2021).

A pandemia da Covid-19 trouxe consigo um cenário desafiador para diferentes setores, como saúde, educação, economia, entre outros. Devido ao aumento de infectados e mortes decorrentes da doença, foram necessárias medidas para conter sua disseminação, a partir do distanciamento social, de restrição do funcionamento de escolas, universidades, transporte público, áreas esportivas e outras instituições, o que acarretou impactos sociais, econômicos e psicológicos para toda a sociedade (ARRUDA EP, 2020; GARCIA LP e DUARTE E, 2020).

$\mathrm{Na}$ área da educação, em especial para o Ensino Superior, foram estabelecidas diretrizes para ampliar a modalidade de ensino à distância de forma emergencial, por meio da Portaria $\mathrm{n}^{\circ} \mathbf{3 4 3}$ de 2020 do Ministério da Educação (MINISTÉRIO DA EDUCAÇÃO, 2020). A partir dessa medida, alunos e professores passaram a usar os meios digitais, tanto em aulas quanto em atividades de extensão, na tentativa de se manterem ativos e atualizados em relação às grades curriculares (GOH PS e SANDARS J, 2020).

Desse modo, as atividades de ensino e aprendizagem necessitaram de importante ressignificação e reformulação, com a implantação da "Educação Remota Emergencial", uma modificação na forma de oferta do conhecimento, com auxílio do ensino remoto para aulas previamente elaboradas para a modalidade presencial (GOH PS e SANDARS J, 2020). Nesse modelo as aulas são transmitidas em tempo instantâneo, de forma síncrona, por meio de sistemas de webconferências (lives), que permitem que professores e alunos tenham condições de interagir e organizar seu tempo de aprendizagem da forma mais próxima à educação presencial (ARRUDA EP, 2020).

Visando atender essa nova realidade, diversas atividades foram adaptadas, incluindo aquelas desenvolvidas pelas Ligas Acadêmicas, que realizavam atividades presenciais e aderiram às estratégias de ensino remoto. Com isso, foi necessária a inclusão de Tecnologias da Informação e Comunicação (TIC), caracterizadas como ferramentas computacionais e meios telecomunicativos que facilitam a difusão das informações por meio de plataformas virtuais (FERRARINI R, et al., 2019). 
As Ligas são entidades universitárias que integram as atividades de ensino, pesquisa e extensão, e têm como objetivo proporcionar discussões aprofundadas aos alunos de graduação sobre assuntos muitas vezes não discutidos em sala de aula. Estas são formadas por alunos de graduação de diferentes anos, tutores e colaboradores e promovem atividades de extensão, troca de conhecimentos e debates que auxiliam na formação acadêmica e profissional dos envolvidos (SILVA DT, et al., 2014; MELO JAC, et al., 2020).

O espaço das Ligas proporciona autonomia para o desenvolvimento habilidades administrativas e burocráticas, além de prover meios para experimentação e reflexões que vão além das aprendizagens didáticas curriculares, ampliando a visão com ações que impactam também a sociedade. Além disso, a liberdade de criação e o protagonismo estudantil promovem responsabilidade, comprometimento, amadurecimento da criticidade e confiança para tomada de decisões, contribuindo para o enfrentamento de situações adversas e liderança (SILVA SA e FLORES O, 2015).

Perante a necessidade de utilizar novas práticas para dar continuidade à atuação da Liga Acadêmica (LA) estudada, da utilização emergencial das TIC no ensino e da reflexão sobre essa vivência, justifica-se este relato, que busca responder ao seguinte questionamento: Quais as dificuldades e facilidades encontradas na atuação da LA durante o ano de 2020, primeiro ano da pandemia de COVID-19, no desenvolvimento das atividades remotas? O objetivo do estudo é relatar a experiência de atuação de uma LA de Cuidados à Saúde da Mulher durante o ano de 2020, apresentando as dificuldades e facilidades encontradas frente às atividades remotas.

\section{RELATO DA EXPERIÊNCIA}

A LA relatada foi fundada em 2013, em uma universidade pública estadual, localizada no interior do estado de São Paulo, Brasil, como modalidade de atividade extracurricular de cultura e extensão universitária e cumpre seu papel realizando diversos eventos direcionados ao público acadêmico e à sociedade, expandindo o conhecimento acerca da temática de saúde da mulher em seu ciclo vital. Seus membros são alunas de graduação em Enfermagem, docentes da área de saúde da mulher e enfermeiras colaboradoras que atuam na área da mulher. Anteriormente à pandemia, eram realizadas reuniões quinzenais para o planejamento e organização dos eventos, além de estabelecer metas a serem alcançadas. No entanto, a partir de março de 2020, as atividades passaram a ser desenvolvidas remotamente.

Frente a isso, entre os meses de março e outubro de 2020, foi necessária a utilização das plataformas digitais que, por meio de acesso a internet em aparelhos eletrônicos, como celulares, computadores e notbooks, permitem a comunicação e a interação entre palestrantes/membros da Diretoria da Liga com os ouvintes de forma instantânea.

O planejamento, a temática e a data de cada evento, sempre foi uma escolha democrática entre os membros da LA. Mesmo de forma remota, isso foi mantido e surgiram novas demandas, como: a escolha da plataforma digital mais adequada; o treinamento dos membros e das palestrantes para o uso dessas plataformas; a preocupação com ataques cibernéticos durante as atividades e com a estabilidade do sinal digital durante as transmissões; e apreender a lidar com um novo modelo de organização. Assim, para transmitir os eventos, a Liga escolheu utilizar o Google Meet $\Theta$, StreamYard $\Theta$, Even $3 \AA$ e YouTube $\AA$ e para divulgá-los optou-se por usar as redes sociais, como o Instagram $\Theta$, Facebook ${ }^{\circledR}$, além do correio eletrônico.

A escolha dos temas de cada evento, ocorreu por meio de sugestões solicitadas aos seguidores das redes sociais e para o público em geral, através de caixas de sugestões e formulários eletrônicos do Google Forms enviados por correio eletrônico, cujas respostas foram discutidas de forma democrática durante as reuniões da Liga. Entre os assuntos abordados, destacaram-se: o exercícios físicos aliados à saúde mental na quarentena, violência contra a mulher, a atuação de enfermeiras na pandemia, a atenção às mulheres que vivem em situação de rua durante esse período, os benefícios de práticas integrativas e complementares para o autocuidado das mulheres em tempos de crise, dentre outros. 
Além disso, a LA manteve sua postura em priorizar palestrantes mulheres, enfermeiras, especialistas com experiência prática e teórica, dando-Ihes liberdade e oportunidade para retratar e abordar o tema da maneira como desejavam. Todavia, atenção especial foi dada para a saúde das mulheres envolvendo a repercussão da pandemia em seu cotidiano. Assim, foram promovidas 10 palestras, uma live em rede social, um Simpósio e treze encontros da Diretoria para planejamento, todos realizados por meio virtual.

Os palestrantes eram das cidades de Campinas, Araraquara, Bauru, Rio de Janeiro, Uberlândia, Brasília, e de outros países, como Colômbia e Estados Unidos da América. Os ouvintes, em geral, foram graduandos de Enfermagem, Terapia Ocupacional, Educação Física, Fisioterapia, Psicologia, Letras, Administração e Medicina, bem como pós-graduandos e docentes de diversas áreas e estados, como Bahia, Ceará, Goiás, Minas Gerais, Paraíba, Pernambuco, Rio de Janeiro, São Paulo, e estrangeiros da Colômbia.

Com isso, evidenciou-se em 2020, um aumento expressivo de participantes nos eventos. Nesse ano, a LA contou com 1547 ouvintes, média de 141 pessoas por evento. Em 2019 e 2018, a média era de 34 e 36 participantes por evento, respectivamente.

Este relato, por utilizar dados secundários provenientes dos eventos promovidos pela LA, dispensa o Termo de Consentimento Livre e Esclarecido e a aprovação de Comitê de Ética em Pesquisa.

\section{DISCUSSÃO}

De modo geral, observa-se que a utilização dos meios digitais favoreceu a LA relatada ao proporcionar a contrução de um espaço de debate e de troca de conhecimentos, atendendo aos seus objetivos. Nesse contexto, considera-se que esse foi um ponto positivo, pois permitiu maior interação da Liga com a comunidade acadêmica e não acadêmica.

Os eventos de uma LA, por se tratarem de atividades extracurriculares, devem buscar discutir temas não abordados na grade curricular ou aprofundar a discussão dos assuntos em diferentes vertentes, de modo a ampliar horizontes e expor novas perspetivas sobre um assunto. Assim, julga-se importante que a LA discutida tenha tratado os assuntos da saúde da mulher envolvendo o contexto atual, a exemplo da violência contra a mulher, abordada devido ao crescente número de casos de agressões físicas, abuso sexual e feminicídio durante o isolamento social (ORGANIZAÇÃO MUNDIAL DA SAÚDE, 2020), visto que a pandemia impactou diretamente, e principalmente, a saúde dessa população, que já pertencem a um grupo vulnerável pelas desigualdades de gênero, sociais, culturais, econômicas e políticas (BRITO L, et al., 2020).

Frente a isso, fica evidente que a LA buscou colaborar com o aprimoramento da formação de enfermeiros e outros profissionais da saúde, bem como reforçar os preceitos estabelecidos pela Política Nacional de Atenção Integral à Saúde da Mulher (PNAISM), enfatizando a importância da perspectiva da integralidade para o cuidado em saúde das mulheres (MINISTÉRIO DA SAÚDE, 2004).

Além disso, foi perceptível que a LA alcançou um público maior, para além de estudantes de Enfermagem. Acredita-se que isso seja decorrente do modelo remoto, que permite a participação de pessoas de diferentes formações, desde que haja interesse e meios de acesso, e também da divulgação dos eventos nas redes sociais, que contribui para que a informação chegue a mais pessoas. Além disso, ao abordar problemáticas atuais de forma aprofundada, a Liga pode ter atraído maior atenção dos ouvintes.

Entretanto, esse aumento de público exige maior responsabilidade no planejamento das ações. Assim, ficou evidente que as atividades da LA foram intensificadas buscando maior variedade de temas de modo a contemplar toda a complexidade do universo feminino, abarcando, de modo convergente, as questões econômicas, sociais e as relações de gênero com os cuidados em saúde frente à uma crise sanitária.

Pontua-se ainda que em relação às vantagens dos eventos virtuais, estes admitem a participação de palestrantes e ouvintes de diferentes cidades, estados e países. No geral, anterior à pandemia, as possibilidades eram mais restritas, quase que exclusivamente à participantes locais, devido aos custos com transporte e estadia, muitas vezes arcados pelos próprios participntes, quando ouvintes, ou pelos membros 
da LA ou com recursos advindos de doações e de patrocinadores, nos casos dos palestrantes, já que a mesma não possui fins lucrativos. Contudo, ao desenvolver eventos online, houve maior diversidade regional, agregando uma importante pluralidade cultural, como percebido também por outros autores (HOFFMANN WP, et al., 2020).

Além disso, destaca-se que visando a autonomia, a valorização e o empoderamento da mulher, a LA priorizou a participação de palestrantes mulheres. Isso é de extrema relevancia, pois os ambientes de extensão podem promover um local de discussões, com a participação feminina de diferentes setores da sociedade, possibilitando a troca de experiências, sentimentos e conflitos, estimulando reflexões e criando espaços acolhedores, principalmente, para as mulheres (VANIN IM e PINHEIRO CC, 2014). Isso contribui para desmistificar tabus que a sociedade impõe para essa população, desde a infância até a vida adulta.

Além disso, as LA podem ser um ambiente transformador, que permite desenvolver o ensino e a pesquisa, promove vínculos entre estudantes, professores e comunidades, e possibilita um cenário diversificado de práticas e de aproximação entre estudantes e comunidade. Entretanto, como toda gestão de ensino, existem dificuldades encontradas também na manutenção de uma LA, como por exemplo, a escassez de tempo disponível para a participação em atividades extracurriculares, referidas assim por ligantes e docentes (SILVA SA e FLORES O, 2015).

Somam-se a isso, os obstáculos provocados pela pandemia da Covid-19, que colocou a comunidade estudantil em isolamento, levando os países a desenvolverem ações de educação remota emergencial. No ensino superior, composto por jovens adultos, houve menos resistência em relação a implementação de tecnologias digitais no processo de ensino aprendizagem. Entretanto, a falta de acesso à internet por uma parcela considerável da população, bem como à equipamentos e dispositivos necessários, como celulares, computadores e notbooks, podem interferir na qualidade das atividades (ARRUDA EP, 2020).

Segundo dados da Organização das Nações Unidas para a Educação, a Ciência e a Cultura (UNESCO), 165 países foram afetados pela pandemia da COVID-19, atingindo cerca de 1,5 bilhão de crianças e jovens, o que representa $87 \%$ dos estudantes do mundo (CASTIONI R, et al., 2021). Apesar disso, na instituição em que a LA relatada desenvolve suas atividades, mais de $98 \%$ dos estudantes possuem acesso à internet e 0 restante obteve auxílio da universidade com recursos de equipamentos que permitiram desenvolver seus afazeres (ARRUDA EP, 2020). Considerando o privilégio de acesso à equipamentos e tecnologias, enfrentar esse problema é promover a equidade, visto que ele atinge, especialmente, estudantes de baixa renda, de minorias étnicas e residentes de regiões mais pobres do país (CASTIONI R, et al., 2021).

Além disso, salienta-se que existe uma dificuldade na adaptação ao ensino remoto, visto que ela exige maior nível de concentração, o que pode dificultar o aprendizado. Em sua maioria, os estudantes encontramse em ambiente domiciliar, nem sempre propício à concentração e apresentam-se mais preocupados com o acúmulo de assuntos para o retorno presencial e com a perda ou o atraso do semestre, desencadeando problemas relacionados ao adoecimento mental (TEIXEIRA LAC, et al., 2021). Nesse sentido, como efeito da pandemia e suas restrições, observa-se um aumento significativo de ansiedade, depressão e estresse entre os estudantes universitários (MAIA BR e DIAS PC, 2020).

Apesar dos desafios, a LA estudada encontrou motivações para se manter ativa durante o ano de 2020, dando continuidade à difusão do conhecimento na área de saúde da mulher. Além disso, as atividades remotas permitem maior flexibilidade de horários e reduz a necessidade de carga horária rígida, em um ambiente dito como exaustivo e cansativo para os estudantes em geral, permitindo a eles 0 acesso às atividades de acordo com a disponibilidade individual (MALTA CP, et al., 2021). Assim, essa nova possibilidade favoreceu os eventos oferecidos pela $L A$, contribuindo para a superação das dificuldades, para a manutenção e maior alcance dos eventos e para a democratização do conhecimento.

É válido salientar também que a participação de qualquer membro de uma LA é uma escolha opcional, o que as torna um espaço teoricamente livre de formalidades acadêmicas, diferente do ambiente hierárquico clássico de relação professor-aluno. Sendo assim, entende-se o aprendizado dentro delas como algo mais prazeroso, favorecendo a saúde mental dos ligantes (SILVA SA e FLORES O, 2015). 
Portanto, a atuação da LA no primeiro ano da pandemia demonstrou ser uma experiência desafiadora e exitosa, que foi possível pela incorporação das TIC e por meio da utilização de plataformas digitais que permitiram manter ativas as atividades desenvolvidas pela LA. Além disso, a facilidade de acessar os eventos por meio virtual permitiu a participação de um público maior e mais variado, levando as discussões e reflexões acerca da saúde das mulheres para qualquer pessoa interessada com condições para acessar os eventos. Isso permitiu desenvolver a extensão universitária, mesmo em ambiente virtual, fortalecendo os pilares universitários, levando conhecimento a estudantes e comunidade, além de aprimorar as discussões acerca da saúde das mulheres com vistas a melhorar a qualidade da saúde.

\section{REFERÊNCIAS}

1. ARRUDA EP. Educação remota emergencial: elementos para políticas públicas na educação brasileira em tempos de Covid-19. EmRede - Revista de Educação a Distância. 2020; 7(1) :257-275.

2. BRITO L, et al. Impactos sociais da Covid-19: uma perspectiva sensível às desigualdades de gênero. Observatório Covid-19 Rio de Janeiro: 2020.2 Disponível em: https://www.arca.fiocruz.br/bitstream/icict/41375/2/ImpactosSociais.PDF. Acessado em 21 de junho de 2021.

3. CASTIONI R, et al. Universidades federais na pandemia da Covid-19: acesso discente à cainternet e ensino remoto emergencial. Ensaio: Avaliação e Políticas Públicas em Educação. 2021; 29(111) :399-419.

4. FERRARINI R, et al. Metodologias ativas e tecnologias digitais: aproximações e distinções. Revista Educação em Questão, 2019; 57(52): 1-30.

5. GARCIA LP, DUARTE E. Intervenções não farmacológicas para o enfrentamento à epidemia da COVID-19 no Brasil. Epidemiologia e Serviços de Saúde, 2020; 29(2): e2020222.

6. GOH PS, SANDARS J. A vision of the use of technology in medical education after the COVID-19 pandemic. MedEdPublish, 2020; 9(1): 49.

7. HOFFMANN WP, et al. A importância do ensino remoto: um relato da Universidade d estado de Mato Grosso. Resrch, Society and Development, 2020; 9(9): e813998084.

8. MAIA BR, DIAS PC. Ansiedade, depressão e estresse em estudantes universitários: o impacto da COVID-19. Estudos de Psicologia (Campinas), 2020; 37(1): e200067.

9. MALTA CP, et al. Atuação da liga acadêmica de cardiologia durante a pandemia da Covid-19 e as implicações na educação médica: relato de experiência. Revista Eletrônica Acervo Saúde, 2021; 13(1): e5588.

10. MELO JAC, et al. A extensão universitária frente ao isolamento social imposto pela COVID-19. Brazialian Journal of Development. 2020; 6(9) :72999-73010.

11. MINISTÉRIO DA EDUCAÇÃO. Portaria no 343, de 17 de março de 2020. Dispõe sobre a substituição das aulas presenciais por aulas em meios digitais enquanto durar a situação de pandemia do Novo Coronavírus - COVID-19. Brasília, DF, Brasil, 2020. Disponível em: https://www.in.gov.br/en/web/dou/-/portaria-n-343-de-17-de-marco-de-2020248564376. Acessado em: 27 de junho de 2021.

12. MINISTÉRIO DA SAÚDE. Política Nacional de Atenção Integral à Saúde da Mulher: princípios e diretrizes. Brasília: Brasil, 2004. Disponível em: https://conselho.saude.gov.br/ultimas_noticias/2007/politica_mulher.pdf. Acessado em: 02 de junho de 2021.

13. ORGANIZAÇÃO DAS NAÇÕES UNIDAS (ONU). Diretrizes para atendimento em casos de violência de gênero contra meninas e mulheres em tempos da pandemia da COVID-19. Brasília: ONU Mulheres. 2020. Disponível em: http://www.onumulheres.org.br/wp-content/uploads/2020/08/Diretrizes-para-atendimento_ONUMULHERES.pdf. Acessado em: 27 de Agosto de 2021.

14. ORGANIZAÇÃO MUNDIAL DA SAÚDE (OMS). WHO Coronavirus Disease (COVID-19) Dashboard. Disponível em: https://covid19. who.int/. Acessado em: 13 de Setembro de 2021.

15. SILVA DT, et al. A evolução do processo ensino - aprendizagem através da implantação da liga acadêmica. Revista Rede de Cuidados em Saúde, 2014; 8(2): 1-4.

16. SILVA SA, FLORES O. Ligas Acadêmicas no Processo de Formação dos Estudantes. Revista Brasileira de Educação Médica, 2015; 39(3): 410-417.

17. TEIXEIRA LAC, et al. Saúde mental dos estudantes de Medicina do Brasil durante a pandemia da coronavirus disease. Jornal Brasileiro de Psiquiatria, 2021; 70(1): 21-29.

18. VANIN IM, PINHEIRO CC. Extensão universitária feminista: espaços de formação para o empoderamento e a equidade de gênero. In: Universidade e sociedade: concepções e projetos de extensão universitária. Salvador: EDUFBA, 2014; 299 p. 